\title{
Surface charge and topography of minerals as a driver for DNA storage and preservation in sediments
}
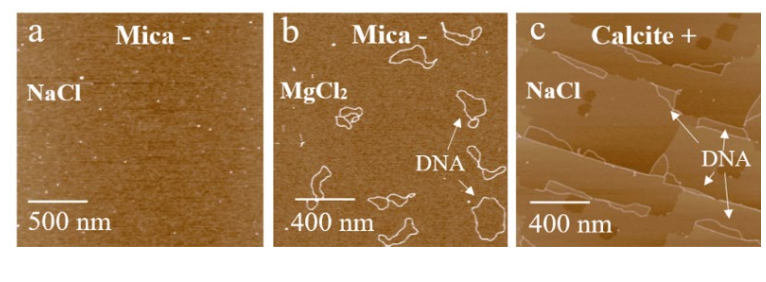

\section{COLIN FREEMAN ${ }^{1}$, LEÁ DIEUDONNÉ ${ }^{2}$, MATTHEW COLLINS $^{3}$ AND KARINA K. KRARUP SAND ${ }^{4}$}

${ }^{1}$ University of Sheffield

${ }^{2}$ École nationale supérieure de chimie de Mulhouse, Université de Haute-Alsace

${ }^{3}$ University of Copenhagen, GLOBE Institute

${ }^{4}$ University of Copenhagen

Presenting Author: kks@sund.ku.dk

DNA stored in sediments (sedimentary ancient DNA, sedaDNA) is increasingly used to provide information on past ecosystems and biodiversity. The field is, however, challenged by a limited understanding of sedaDNA-sediment interactions and it is currently unclear how and under which conditions minerals facilitate preservation of the sedaDNA across time and space.

From the standpoint of Considering adsorption theory, the electrostatics of the DNA-mineral-solution interface will drive adsorption and influence DNA stability. Here we investigated DNA adsorption to a positively and a negatively charged mineral surface in a range of environmentally relevant solution compositions. We used atomic force microscopy to study DNA adsorption behavior as a function of solution composition and molecular dynamics simulations to explore the mechanistic level information associated with binding.

We find that the surface composition and local charges determine the nature of the DNA-mineral interaction. Further, we show that the binding to the surface can facilitate fragmentation, an interesting result given that a common characteristic of mineral associated DNA (ancient sediment and skeletons) is its short fragment length. Combined the results highlight that knowledge of the mineralogical composition of a sediment and the environmental conditions can be useful for both assessing the sedaDNA adsorption and preservation potential.

Figure 1. Atomic force microscopy images showing conformations of a circular DNA (white parts in images) when bound to 2 differently charged minerals a and b) mica and c) calcite. Mica is negatively charged and adsorption of the negatively charged DNA strand is dependent on a charge dense cation (i.e. $\mathrm{Mg}^{2+}$ ). In the absence of such a cation the DNA is easily desorbed as seen in a). Calcite is positively charged and its steps (edges in c) have a high charge density. There is a strong preference for DNA binding on the steps and they retain DNA regardless of the cations present (illustrated by the binding with the low charge dense cation $\mathrm{Na}^{+}$). The DNA is subsequently difficult to desorb.[1]

\section{References}

[1] Freeman, Dieudonne,Collins, ; Sand, K. K. Survival of Environmental DNA in Natural Environments: Surface Charge and Topography of Minerals as Driver for DNA Storage. bioRxiv 2020, doi.org/10.1101/2020.01.28.922997. 